Article

\title{
Evaluation of a Lateral Flow Assay for Rapid Detection of African Swine Fever Virus in Multiple Sample Types
}

\author{
Chukwunonso Onyilagha ${ }^{1}$, Kelvin Nguyen ${ }^{1}$, Pam D. Luka ${ }^{2}$ (D), Ularamu Hussaini ${ }^{2}$, Adeyinka Adedeji ${ }^{2}$ (D), \\ Theophilus Odoom ${ }^{3}$ and Aruna Ambagala 1,4,*(D)
}

1 National Centre for Foreign Animal Disease, Canadian Food Inspection Agency, Winnipeg, MB R3E 3M4, Canada; chukwunonso.onyilagha@inspection.gc.ca (C.O.); nguyenk9@myumanitoba.ca (K.N.)

2 Virology Division, National Veterinary Research Institute, P.M.B 01, Vom 930001, Plateau, Nigeria; pamluka08@gmail.com (P.D.L.); ularamuhussaini@yahoo.co.uk (U.H.); yinkadeji@yahoo.com (A.A.)

3 Accra Laboratory, Veterinary Services Directorate, Accra P.O. Box M161, Ghana; theodoom@yahoo.com

4 Department of Comparative Biology, Faculty of Veterinary Medicine, University of Calgary, Calgary, AB T2N 1N4, Canada

* Correspondence: aruna.ambagala@inspection.gc.ca; Tel.: +1-204-789-2013

check for

updates

Citation: Onyilagha, C.; Nguyen, K.; Luka, P.D.; Hussaini, U.; Adedeji, A.; Odoom, T.; Ambagala, A. Evaluation of a Lateral Flow Assay for Rapid Detection of African Swine Fever Virus in Multiple Sample Types. Pathogens 2022, 11, 138. https:// doi.org/10.3390/pathogens11020138

Academic Editor:

Marta Martínez-Avilés

Received: 21 December 2021

Accepted: 19 January 2022

Published: 24 January 2022

Publisher's Note: MDPI stays neutral with regard to jurisdictional claims in published maps and institutional affiliations.

Copyright: (C) 2022 by the authors. Licensee MDPI, Basel, Switzerland. This article is an open access article distributed under the terms and conditions of the Creative Commons Attribution (CC BY) license (https:// creativecommons.org/licenses/by/ $4.0 /)$.

\begin{abstract}
Antibody-based lateral flow assay (LFA) is a quick and inexpensive tool used to detect pathogens in field samples, especially in hard-to-reach remote areas that may have limited access to central laboratories during an outbreak or surveillance. In this study, we investigated the ability of a commercially available LFA, PenCheck ${ }^{\circledR}$, to detect African swine fever virus (ASFV) in clinical samples derived from pigs infected with highly virulent ASFV strains. The assay was specific and positively identified the majority of pigs showing high fever during the early stages (between 3 and 5 days) of infection. PenCheck ${ }^{\circledR}$ LFA also detected ASFV in serum and tissue samples collected from pigs that succumbed to experimental ASFV infection and whole blood, plasma, and tissue samples from the field. The limit of detection of the assay was ASFV titer $10^{7.80} \mathrm{TCID}_{50} / \mathrm{mL}$, corresponding to ASFV real-time PCR values below $23 \mathrm{Ct}$. Although the sensitivity of the assay is less than that of the laboratory-based real-time PCR assays, the results obtained with the PenCheck ${ }^{\circledR}$ LFA in this study suggest that it can be used as a herd-level, field-deployable, and easy-to-use diagnostic tool to identify ASF-affected farms when access to portable molecular assays or central laboratories is not possible.
\end{abstract}

Keywords: African swine fever; lateral flow assay; PenCheck; real-time PCR

\section{Introduction}

African swine fever (ASF) remains a global priority transboundary animal disease. It is a highly fatal hemorrhagic disease of domestic and wild swine that continues to spread across Africa, Europe, Asia, and the Caribbean [1-9]. The causative agent, ASF virus (ASFV), is a structurally complex, large double-stranded DNA virus and the sole member of the Asfaviridae family belonging to the genus Asfivirus [10]. ASF, a reportable disease to the World Organization for Animal Health (OIE), can cause up to $100 \%$ mortality depending on the strain. The transmission of ASFV can occur through direct contact between infected and uninfected pigs or indirectly through contaminated meat products, fomites, or tick bites [11]. Until recently, ASF remained endemic in Africa [12,13] and Sardinia [7,14]; however, in 2007, ASF spread to Georgia, then to Russia, eastern and western Europe, Southeast Asia, and the Caribbean, killing millions of pigs with accompanying severe economic losses $[9,11,13,15-17]$. There is currently no commercially available approved vaccine for ASF [18,19]. Therefore, early detection is critical for controlling the spread of the virus. Real-time PCR is the most widely used method for detecting ASFV genomic material in clinical samples [20]. The current real-time PCR assays are highly sensitive and specific, 
but they are generally laborious, expensive, and require trained personnel in the laboratory to conduct the testing. Other molecular assays, including portable real-time PCR assays and loop-mediated isothermal amplification (LAMP) assays, have been developed for field testing; however, they still demand some technical competencies at the field level as they require nucleic acid extraction and pipetting [21-31].

Lateral flow assays (LFAs), in contrast, can easily be performed by following simple instructions, and are thus ideal for field use. Many LFAs have been developed and commercialized for pen/bedside use to detect biomarkers, viral and bacterial antigens in various clinical sample types like whole blood, serum, plasma, urine, and saliva [32-35]. Recently, an antibody-based LFA, PenCheck ${ }^{\circledR}$ (Silver Lake Research, CA, USA), was developed and commercialized in the USA for portable detection of ASF. Here, we describe the evaluation of PenCheck ${ }^{\circledR}$ LFA for its ability to detect African swine fever virus (ASFV) in clinical samples collected from pigs infected with highly virulent ASFV strains. PenCheck ${ }^{\circledR}$ LFA targets ASFV P30, a highly antigenic, secreted phosphoprotein expressed during the early stages of ASFV infection [36].

\section{Results and Discussion}

There are highly sensitive and specific molecular assays available for ASFV genome detection. Most of these assays demand sophisticated and expensive instrumentation and highly skilled staff; therefore, they are mostly limited to laboratory use. In recent years, some of these laboratory-based molecular assays have been adapted for use on portable devices $[37,38]$. Novel assays, such as LAMP, clustered regularly interspaced short palindromic repeats (CRISPR)/Cas12a, and recombinase polymerase amplification (RPA) assays, have been developed for field detection of ASF [21-31]. These assays, however, can be costly and demand some technical expertise. Therefore, portable molecular assays may not be useful for ASF diagnosis in poor resource settings.

Antibody-based LFAs, in contrast, are relatively cheap, require no additional instrumentation, and can easily be performed by anyone following simple instructions. They are one-time-use assays and pose no risk of transmitting pathogens between farms when used during an outbreak $[25,26,39]$. In general, antibody-based LFAs are less sensitive compared with their molecular counterpart assays. However, as pigs infected with highly virulent ASFV strains develop high viral titers in blood within a few days after infection, antibody-based LFAs could be useful for rapidly detecting ASFV-infected pigs during the early stages of the infection. Therefore, we wanted to determine the suitability of the commercially available antibody-based LFA, PenCheck ${ }^{\circledR}$, for early detection of ASFV in pigs infected with highly virulent ASFV strains.

Towards this goal, we first determined the analytical sensitivity of the PenCheck ${ }^{\circledR}$ LFA using a dilution series of whole blood samples collected from two pigs infected with highly virulent ASFV strains, ASFV Georgia 2007/1 (Pig \#134) or ASFV Nigeria RV502 (Pig \#2). For comparison, the serial dilutions were also subjected to real-time PCR and virus titration. PenCheck ${ }^{\circledR}$ LFA detected ASFV in undiluted and tenfold-diluted whole blood samples (Table $1(\mathrm{~A}, \mathrm{~B})$ ) for both viruses, translating to $\mathrm{Ct}$ values of $<23$ and virus titers $\geq 10^{7.80}$ (Table 1 (A)) and $\geq 10^{7.89}$ (Table 1 (B)) TCID $50 / \mathrm{mL}$. To assess the analytical specificity of the PenCheck ${ }^{\circledR}$ LFA, we used archived cell culture-amplified ASFV p72 genotype I strains (ASFV Malta'78, ASF OURT/88/3, and ASFV Malawi LIL 18/2) and whole blood and tissue (spleen) samples collected from pigs infected with three different classical swine fever virus (CSFV) strains, as well as other cell culture-amplified porcine viruses. PenCheck ${ }^{\circledR}$ LFA detected all ASFV p72 genotype I strains tested and did not cross-react with any tested classical swine fever (CSF), vesicular stomatitis, foot-and-mouth disease, swine vesicular disease, and Seneca valley virus strains (Table $1(\mathrm{C})$ ). To evaluate the reproducibility of the assay, three known ASFV real-time PCR-positive samples (pig \#140, \#141, and \#145, infected with ASFV Georgia 2007/1) and one known negative (ASFV uninfected) whole blood sample were tested ten times using the PenCheck ${ }^{\circledR}$ LFA. The results were consistent across the samples assessed (Table 1 (D)). The real-time PCR-negative (clean blood) and 
positive (with a Ct value of 23.6) whole blood samples were not detected by PenCheck ${ }^{\circledR}$ LFA, consistent with our earlier observation.

Table 1. Sensitivity, specificity, and reproducibility of the PenCheck ${ }^{\circledR}$ LFA.

\begin{tabular}{|c|c|c|c|c|c|c|c|}
\hline \multicolumn{8}{|c|}{$\mathbf{A}$} \\
\hline \multirow{2}{*}{ ASFV Georgia 2007/1, Pig 134} & \multicolumn{2}{|c|}{ Ct Value (Run 1) } & \multicolumn{2}{|c|}{ Ct Value (Run 2) } & \multirow{2}{*}{$\begin{array}{l}\text { PenCheck } \\
\text { (Run 1) }\end{array}$} & \multirow{2}{*}{$\begin{array}{l}\text { PenCheck } \\
\text { (Run 2) }\end{array}$} & \multirow{2}{*}{$\begin{array}{l}\text { ASFV Titer } \\
\left.\text { (TCID }_{50} / \mathrm{mL}\right)\end{array}$} \\
\hline & ASFV & $\beta$-Actin & ASFV & $\beta$-Actin & & & \\
\hline Neat & 19.22 & 23.43 & 20.08 & 23.12 & Pos & Pos & $10^{8.87}$ \\
\hline $10^{-1}$ & 22.63 & 24.67 & 22.66 & 24.88 & Pos & Pos & $10^{7.80}$ \\
\hline $10^{-2}$ & 25.64 & 26.01 & 25.57 & 26.34 & Neg & Neg & $10^{6.71}$ \\
\hline $10^{-3}$ & 28.24 & 25.23 & 27.88 & 25.95 & Neg & Neg & nd \\
\hline $10^{-4}$ & 30.89 & 26.34 & 31.21 & 26.29 & Neg & Neg & nd \\
\hline \multicolumn{8}{|c|}{ B } \\
\hline \multirow{2}{*}{ ASFV Nigeria RV502, Pig 2} & \multicolumn{2}{|c|}{ Ct Value (Run 1) } & \multicolumn{2}{|c|}{ Ct Value (Run 2) } & \multirow{2}{*}{$\begin{array}{l}\text { PenCheck } \\
\text { (Run 1) }\end{array}$} & \multirow{2}{*}{$\begin{array}{l}\text { PenCheck } \\
\text { (Run 2) }\end{array}$} & \multirow{2}{*}{$\begin{array}{l}\text { ASFV Titer } \\
\text { (TCID50/mL) }\end{array}$} \\
\hline & ASFV & $\beta$-Actin & ASFV & $\beta$-Actin & & & \\
\hline Neat & 19.42 & 22.22 & 18.66 & 22.02 & Pos & Pos & $10^{9.1}$ \\
\hline $10^{-1}$ & 22.01 & 24.63 & 22.01 & 24.54 & Pos & Pos & $10^{7.89}$ \\
\hline $10^{-2}$ & 25.11 & 26.90 & 24.82 & 26.61 & Neg & Neg & $10^{6.80}$ \\
\hline $10^{-3}$ & 27.71 & 27.36 & 27.23 & 27.16 & Neg & Neg & $10^{4.87}$ \\
\hline $10^{-4}$ & 29.52 & 26.71 & 29.09 & 26.54 & Neg & Neg & $10^{3.87}$ \\
\hline \multicolumn{8}{|c|}{ C } \\
\hline \multicolumn{5}{|c|}{ Sample } & \multirow{2}{*}{\multicolumn{2}{|c|}{$\frac{\text { Ct Value }}{\text { nd }}$}} & PenCheck \\
\hline ASFV Malta'78 & & & & & & & Pos \\
\hline ASFV OURT $/ 88 / 3$ & & & & & & nd & Pos \\
\hline ASFV Malawi LIL 18/2 & & & & & & nd & Pos \\
\hline CSFV Kanagawa, P2, DPI 42 & & & & & & 16.0 & Neg \\
\hline CSFV Kanagawa, P4, DPI 42 & & & & & & 19.4 & Neg \\
\hline CSFV Diepholz, P3, DPI 8-2 & & & & & & 22.1 & $\mathrm{Neg}$ \\
\hline CSFV Diepholz, P1, DPI 12-6 & & & & & & 22.1 & Neg \\
\hline CSFV Diepholz, P2, DPI 12-6 & & & & & & 20.5 & $\mathrm{Neg}$ \\
\hline CSFV Diepholz, P3, DPI 12-6 & & & & & & 21.1 & $\mathrm{Neg}$ \\
\hline CSFV Diepholz, P4, DPI 12-6 & & & & & & 22.2 & $\mathrm{Neg}$ \\
\hline CSFV Koslov, P5, DPI 6-8, Spleen & & & & & & 16.4 & Neg \\
\hline CSFV Koslov, P7, DPI 6-8, Spleen & & & & & & 18.4 & Neg \\
\hline CSFV Koslov, P8, DPI 6-8, Spleen & & & & & & 17.6 & Neg \\
\hline Vesicular Stomatitis Virus-NJ & & & & & & 15.01 & Neg \\
\hline Vesicular Stomatitis Virus-IN & & & & & & 15.29 & Neg \\
\hline Foot-and-mouth Disease Virus OL & G $11 / 01$ & & & & & 12.36 & Neg \\
\hline Foot-and-mouth disease virus A2 & rruzeiro & & & & & 11.99 & Neg \\
\hline Swine Vesicular Disease Virus UK & $/ 72$ & & & & & 11.61 & Neg \\
\hline Seneca Valley Virus & & & & & & 12.7 & $\mathrm{Neg}$ \\
\hline & & & D & & & & \\
\hline
\end{tabular}

\begin{tabular}{ccccc}
\hline \multicolumn{7}{c}{ PenCheck: ASFV Georgia 2007/1 } \\
\hline Pig 140, DPI-12 (Ct: 23.6) & Pig 141, DPI-12 (Ct: 19.9) & Pig 145, DPI-12 (Ct: 21.0) & Clean Blood (Ct: 0.0) \\
\hline Run 1 & Neg & Pos & Pos & Neg \\
\hline Run 2 & Neg & Pos & Pos & Neg \\
\hline Run 3 & Neg & Pos & Pos & Neg \\
\hline Run 4 & Neg & Pos & Pos & Neg \\
\hline Run 5 & Neg & Pos & Pos & Neg \\
\hline Run 6 & Neg & Pos & Pos & Neg \\
\hline Run 7 & Neg & Pos & Pos & Neg \\
\hline Run 8 & Neg & Pos & Pos & Neg \\
\hline Run 9 & Neg & Pos & Pos & Neg \\
\hline Run 10 & Neg & Pos & Pos & \\
\hline
\end{tabular}

The analytical sensitivity of the assay was measured using serial dilution of blood samples collected from pigs infected with ASFV Georgia 2007/1 (A) or ASFV Nigeria RV502 (B). The specificity of the PenCheck ${ }^{\circledR}$ LFA was measured using cell culture-amplified ASFV strains (genotype I) and whole blood and tissue samples from pigs infected with classical swine fever virus strains, as well as cell culture-amplified vesicular stomatitis virus strains, foot-and-mouth disease virus strains, swine vesicular disease virus, and Seneca valley virus (C). Reproducibility of the assay was measured using blood samples collected from three pigs infected with ASFV Georgia 2007/1 (D) For $(\mathrm{C})$, the $\beta$-actin Ct value range for the samples tested by real-time PCR was 19.4 to 25.1. Pos, positive (green); Neg, negative (yellow); nd, not done.

Currently, there is no effective vaccine for ASF; therefore, rapid detection, preferably on-site, and removal of the infected and contact pigs (stamping out) is the most effective 
approaches to curtail the spread of the disease. Highly virulent strains of ASFV have an incubation period of 2-3 days; fever, lack of appetite, and lethargy are the first clinical signs to appear. Fever in ASFV-infected animals coincides with viremia, which quickly peaks (within 1-2 days of fever) up to $10^{8-9} \mathrm{TCID}_{50} / \mathrm{mL}$ [40,41]. Therefore, despite the low sensitivity of the PenCheck ${ }^{\circledR}$ LFA compared with real-time PCR (see Table 1 (A,B)), we wanted to determine if the PenCheck ${ }^{\circledR}$ LFA can be used to detect ASFV in whole blood samples collected from pigs showing early clinical signs of ASF. For this, we used whole blood samples collected from pigs directly or indirectly (contact with a directly infected pig) infected with two different strains of highly virulent ASFV (ASFV Georgia $2007 / 1$ and ASFV Nigeria RV502) belonging to p72 genotype II. The PenCheck ${ }^{\circledR}$ LFA results were compared with the real-time PCR results and rectal temperatures. Pigs with rectal temperatures above $40^{\circ} \mathrm{C}$ for two consecutive days were considered as having a fever.

The first set of whole blood samples tested were collected from ASFV Georgia 2007/1 infected pigs (Figure S2). Whole blood samples collected from a seeder pig (\#138) that was directly infected with ASFV Georgia 2007/1 tested positive on PenCheck ${ }^{\circledR}$ LFA starting from $4 \mathrm{dpi}$, the same day as the onset of fever (real-time PCR Ct value of 21.6) (Table 2). The seeder pig remained positive on PenCheck ${ }^{\circledR}$ LFA until it was found dead on 7 dpi. Four contact pigs (\#126, 129, 139, and 141) started to develop fever 10 days post-exposure (dpe); two of them (\#139 and 141) tested positive on PenCheck ${ }^{\circledR}$ LFA on 12 dpe. Three additional pigs (\#144, 145, and 146) started to develop fever on 12 dpe; in addition to pigs \#139 and 141 , two of those three pigs (\#145 and 146) tested positive on PenCheck ${ }^{\circledR}$ LFA on 12 dpe. On 13 dpe, two more pigs (\#130 and 140) developed fever, and three pigs (\#126, 132 and 134) tested positive on PenCheck ${ }^{\circledR}$ LFA. The remaining four pigs that developed fever tested positive on PenCheck ${ }^{\circledR}$ LFA on 14 (pigs \#140 and 144) and 15 dpe (pigs \#129 and 130). One contact pig (\#133) remained uninfected (based on Ct value and fever data) and tested negative on PenCheck ${ }^{\circledR}$ LFA until the termination of the experiment on $17 \mathrm{dpe}$. The remaining animals tested positive on PenCheck ${ }^{\circledR}$ LFA at least once during the study when their whole blood Ct values fell below 23. Two samples (pig \#130, 17 dpe and pig \#134, 16 dpe), however, repeatedly tested negative on PenCheck ${ }^{\circledR}$ LFA despite having low Ct values (20.9 and 21.6, respectively). In order to determine the cause of the negative results, the viral titers of these two samples were determined to be $10^{9.10}$ and $10^{5.87} \mathrm{TCID}_{50} / \mathrm{mL}$ for pigs \#130 on 17 dpe and pig \#134 on 16 dpe, respectively. While the low ASFV titer in the sample from pig \#134 could account for the negative results obtained with PenCheck ${ }^{\circledR}$ LFA, the same could not be said of the sample from pig \#130. We speculate that the discordance could be due to additional factors specific to these two samples.

In pigs, rectal temperature starting at $40.5{ }^{\circ} \mathrm{C}$ is considered a high fever, and at this point, they often become lethargic and show less interest in feeding. All pigs that developed a high fever for two consecutive days in this experiment had whole blood $\mathrm{Ct}$ values below 23, and when tested, they were identified as ASF positive by the PenCheck ${ }^{\circledR}$ LFA.

To further evaluate PenCheck ${ }^{\circledR}$ LFA, we used whole blood and tissue samples collected from two pigs simultaneously infected with ASFV Nigeria RV502, an isolate obtained from the 2020 ASF p72 genotype II outbreak in Nigeria (Table 3). Of the two pigs, \#2 started to develop fever on $3 \mathrm{dpi}$, and \#1 on $4 \mathrm{dpi}$. Pig \#1 tested positive by real-time PCR on $3 \mathrm{dpi}$ and $\# 2$ on 4 dpi. Both animals tested positive on PenCheck ${ }^{\circledR}$ LFA on 4 dpi, when they developed a high fever $\left(\geq 40.5^{\circ} \mathrm{C}\right)$ and remained positive until they were found dead (pig \#1) or euthanized (pig \#2) on 6 dpi. When pig \#2 tested positive on PenCheck ${ }^{\circledR}$ LFA, the whole blood sample had a $\mathrm{Ct}$ value of 27.12. The same sample was tested multiple times on PenCheck ${ }^{\circledR}$ LFA and by real-time PCR, and the results remained the same. 
Table 2. Comparing PenCheck ${ }^{\circledR}$ LFA results with the onset of clinical sign (fever) and detection of ASFV genomic material in ASFV Georgia 2007/1-infected pigs.

\begin{tabular}{|c|c|c|c|c|c|c|c|c|c|c|c|c|c|c|c|c|c|c|c|c|c|c|c|c|c|c|c|c|c|}
\hline & & DPI 2 & & & $\overline{\text { DPI } 4}$ & & & DPI 5 & & & DPI 6 & & & & & & & & & & & & & & & & & & \\
\hline $\begin{array}{c}\text { Pig } \\
\#\end{array}$ & Temp. & $\mathrm{Ct}$ & $\begin{array}{l}\text { Pen } \\
\text { Check }\end{array}$ & Temp. & $\mathrm{Ct}$ & $\begin{array}{l}\text { Pen } \\
\text { Check }\end{array}$ & Temp. & $\mathrm{Ct}$ & $\begin{array}{l}\text { Pen } \\
\text { Check }\end{array}$ & Temp. & $\mathrm{Ct}$ & $\begin{array}{l}\text { Pen } \\
\text { Check }\end{array}$ & & & & & & & & & & & & & & & & & \\
\hline \multirow[t]{2}{*}{$\begin{array}{l}\text { Pig } \\
138 \\
\end{array}$} & na & 38.1 & Neg & 40.8 & 21.6 & Pos & 40.6 & 21.1 & Pos & 40.8 & 20.6 & Pos & & & & & & & & & & & & & & & & & \\
\hline & \multicolumn{3}{|c|}{ DPE 8} & \multicolumn{3}{|c|}{$\overline{\text { DPE } 9}$} & \multicolumn{3}{|c|}{$\overline{\text { DPE } 10}$} & \multicolumn{3}{|c|}{ DPE 11} & \multicolumn{3}{|c|}{ DPE 12} & \multicolumn{3}{|c|}{$\overline{\text { DPE } 13}$} & \multicolumn{3}{|c|}{ DPE 14} & \multicolumn{3}{|c|}{ DPE 15} & \multicolumn{3}{|c|}{ DPE 16} & \multicolumn{2}{|c|}{ DPE 17} \\
\hline $\begin{array}{c}\text { Pig } \\
\#\end{array}$ & Temp. & $\mathrm{Ct}$ & $\begin{array}{l}\text { Pen } \\
\text { Check }\end{array}$ & Temp. & $\mathrm{Ct}$ & $\begin{array}{l}\text { Pen } \\
\text { Check }\end{array}$ & Temp. & $\mathrm{Ct}$ & $\begin{array}{l}\text { Pen } \\
\text { Check }\end{array}$ & Temp. & $\mathrm{Ct}$ & $\begin{array}{l}\text { Pen } \\
\text { Check }\end{array}$ & Temp. & $\mathrm{Ct}$ & $\begin{array}{l}\text { Pen } \\
\text { Check }\end{array}$ & Temp. & $\mathrm{Ct}$ & $\begin{array}{l}\text { Pen } \\
\text { Check }\end{array}$ & Temp. & $\mathrm{Ct}$ & $\begin{array}{l}\text { Pen } \\
\text { Check }\end{array}$ & Temp. & $\mathrm{Ct}$ & $\begin{array}{l}\text { Pen } \\
\text { Check }\end{array}$ & Temp. & . $\mathrm{Ct}$ & $\begin{array}{c}\text { Pen } \\
\text { Check }\end{array}$ & Temp. Ct & $\begin{array}{l}\text { Pen } \\
\text { Check }\end{array}$ \\
\hline $\begin{array}{l}\text { Pig } \\
126\end{array}$ & 39.7 & na & na & 39.7 & 0.0 & $\mathrm{Neg}$ & 40.0 & na & na & 40.4 & 28.9 & $\mathrm{Neg}$ & 40.9 & na & na & 40.5 & 20.1 & Pos & 41.3 & na & na & & & & & & & & \\
\hline $\begin{array}{l}\text { Pig } \\
129 \\
\end{array}$ & 39.8 & na & na & 39.6 & 0.0 & Neg & 40.0 & na & na & 40.1 & 0.0 & $\mathrm{Neg}$ & 39.9 & na & na & 41.6 & 25.3 & $\mathrm{Neg}$ & 41.2 & na & na & 41.6 & 20.9 & Pos & 41.6 & na & na & 40.7 & \\
\hline $\begin{array}{l}\text { Pig } \\
132\end{array}$ & 39.6 & na & na & 38.9 & 0.0 & Neg & 39.0 & na & na & 39.9 & 24.2 & Neg & 41.4 & na & na & 41.3 & 19.8 & Pos & & & & & & & & & & & \\
\hline $\begin{array}{l}\text { Pig } \\
133\end{array}$ & 39.5 & na & na & 39.1 & na & na & 39.7 & na & na & 39.7 & na & na & 39.8 & na & na & 39.8 & 0.0 & $\mathrm{Neg}$ & 39.6 & na & na & 39.9 & 0.0 & $\mathrm{Neg}$ & 39.7 & na & na & 40.1 & Neg \\
\hline $\begin{array}{l}\text { Pig } \\
134 \\
\end{array}$ & 39.5 & na & na & 39.6 & na & na & 39.6 & na & na & 39.8 & 30.7 & $\mathrm{Neg}$ & 41.5 & na & na & 41.7 & 19.0 & Pos & 42.2 & na & na & 42.0 & 22.4 & Neg & 41.6 & 21.6 & Neg & & \\
\hline $\begin{array}{l}\text { Pig } \\
139\end{array}$ & 39.9 & 0.0 & Neg & 39.6 & na & na & 40.2 & 31.9 & $\mathrm{Neg}$ & 40.4 & na & na & 41.2 & 20.6 & Pos & 41.6 & na & na & 41.3 & 21.0 & Pos & & & & & & & & \\
\hline $\begin{array}{l}\text { Pig } \\
140\end{array}$ & 39.5 & na & na & 38.8 & na & na & 39.3 & na & na & 39.7 & na & na & 39.5 & 23.6 & $\mathrm{Neg}$ & 41.3 & na & na & 41.4 & 20.3 & Pos & & & & & & & & \\
\hline $\begin{array}{l}\text { Pig } \\
141 \\
\end{array}$ & 40.0 & 0.0 & $\mathrm{Neg}$ & 39.3 & na & na & 40.7 & 26.2 & $\mathrm{Neg}$ & 40.6 & na & na & 41.4 & 19.9 & Pos & 41.0 & 19.8 & Pos & na & na & na & & & & & & & & \\
\hline $\begin{array}{l}\text { Pig } \\
145 \\
\end{array}$ & 39.6 & na & na & 39.1 & na & na & 39.9 & 35.5 & $\mathrm{Neg}$ & 40.5 & na & na & 41.0 & 21.0 & Pos & 40.5 & & & & & & & & & & & & & \\
\hline $\begin{array}{l}\text { Pig } \\
146\end{array}$ & 39.5 & na & na & 38.8 & na & na & 39.8 & 24.9 & Neg & 41.4 & na & na & 40.8 & 19.7 & Pos & 41.0 & na & na & 40.8 & 21.7 & Pos & 41.3 & & & & & & & \\
\hline
\end{tabular}

In a recent ASF transmission study, one pig (\#138) was directly inoculated (intramuscularly) with ASFV Georgia 2007/1 and introduced into a pen with healthy pigs. The whole blood samples collected every other day from the animals that developed clinical signs were tested by real-time PCR and the PenCheck ${ }^{\circledR}$ LFA. Pos, positive (green); Neg, negative (yellow); Temp., temperature; na, not available (gray). The black cells signify the dpe where the animals died. 
Table 3. Comparing PenCheck ${ }^{\circledR}$ LFA results with the onset of clinical sign (fever) and detection of ASFV genomic material in ASFV Nigeria RV502-infected pigs.

\begin{tabular}{|c|c|c|c|c|c|c|c|c|c|c|c|c|c|c|c|c|c|c|c|c|c|c|}
\hline \multicolumn{23}{|c|}{ A } \\
\hline \multicolumn{3}{|c|}{ DPI 0} & \multicolumn{4}{|c|}{ DPI 1} & \multicolumn{3}{|c|}{ DPI 2} & \multicolumn{3}{|c|}{ DPI 3} & \multicolumn{3}{|c|}{ DPI 4} & \multicolumn{3}{|c|}{ DPI 5} & \multicolumn{4}{|c|}{ DPI 6} \\
\hline Sample & Pig \# & Temp. & $\mathrm{Ct}$ & $\begin{array}{l}\text { Pen } \\
\text { Check }\end{array}$ & Temp & $\mathrm{Ct}$ & $\begin{array}{l}\text { Pen } \\
\text { Check }\end{array}$ & Temp & $\mathrm{Ct}$ & $\begin{array}{l}\text { Pen } \\
\text { Check }\end{array}$ & Temp & $\mathrm{Ct}$ & $\begin{array}{l}\text { Pen } \\
\text { Check }\end{array}$ & Temp & $\mathrm{Ct}$ & $\begin{array}{c}\text { Pen } \\
\text { Check }\end{array}$ & Temp & $\mathrm{Ct}$ & $\begin{array}{c}\text { Pen } \\
\text { Check }\end{array}$ & Temp & $\mathrm{Ct}$ & $\begin{array}{c}\text { Pen } \\
\text { Check }\end{array}$ \\
\hline Temperature & Pig 1 & 39.5 & na & na & 39.3 & na & na & 39.5 & na & na & 39.6 & na & na & 40.6 & na & na & 41.3 & na & na & na & na & na \\
\hline \multirow{2}{*}{ Whole blood } & Pig 1 & na & 0.00 & Neg & na & 0.00 & $\mathrm{Neg}$ & na & 0.00 & $\mathrm{Neg}$ & na & 30.81 & $\mathrm{Neg}$ & na & 21.10 & Pos & na & 19.37 & Pos & na & na & na \\
\hline & Pig 2 & na & 0.00 & Neg & na & 0.00 & $\mathrm{Neg}$ & na & 0.00 & $\mathrm{Neg}$ & na & 0.00 & $\mathrm{Neg}$ & na & 27.12 & Pos & na & 21.07 & Pos & na & 20.86 & Pos \\
\hline \multirow[b]{2}{*}{ Nasal swab } & Pig 1 & na & 0.00 & Neg & na & 35.40 & $\mathrm{Neg}$ & na & 0.00 & $\mathrm{Neg}$ & na & 31.80 & $\mathrm{Neg}$ & na & 29.35 & $\mathrm{Neg}$ & na & 22.58 & Neg & na & 25.76 & $\mathrm{Neg}$ \\
\hline & Pig 2 & na & 0.00 & Neg & na & 0.00 & Neg & na & 0.00 & Neg & na & 0.00 & Neg & na & 35.39 & $\mathrm{Neg}$ & na & 27.50 & Neg & na & 24.26 & Neg \\
\hline \multirow{2}{*}{ Oral swab } & Pig 1 & na & 0.00 & Neg & na & 0.00 & $\mathrm{Neg}$ & na & 0.00 & $\mathrm{Neg}$ & na & 0.00 & $\mathrm{Neg}$ & na & 31.82 & Neg & na & 28.85 & $\mathrm{Neg}$ & na & 25.09 & $\mathrm{Neg}$ \\
\hline & Pig 2 & na & 0.00 & Neg & na & 0.00 & Neg & na & 0.00 & Neg & na & 0.00 & $\mathrm{Neg}$ & na & 34.64 & Neg & na & 31.07 & $\mathrm{Neg}$ & na & 24.04 & Neg \\
\hline Rectal swab & Pig 2 & na & 0.00 & Neg & na & 0.00 & $\mathrm{Neg}$ & na & 0.00 & $\mathrm{Neg}$ & na & 0.00 & Neg & na & na & Neg & na & 29.48 & $\mathrm{Neg}$ & na & 24.32 & $\mathrm{Neg}$ \\
\hline \multirow[b]{2}{*}{ Serum } & Pig 1 & na & 0.00 & Neg & na & 0.00 & Neg & na & 0.00 & $\mathrm{Neg}$ & na & 0.00 & $\mathrm{Neg}$ & na & 21.01 & Pos & na & na & na & na & na & na \\
\hline & Pig 2 & na & 0.00 & Neg & na & 0.00 & Neg & na & 0.00 & Neg & na & 0.00 & Neg & na & 30.43 & Neg & na & na & na & na & 18.74 & Pos \\
\hline \multicolumn{23}{|c|}{ B } \\
\hline \multirow{2}{*}{\multicolumn{13}{|c|}{ Sample }} & \multicolumn{5}{|c|}{ Ct Value } & \multirow{2}{*}{\multicolumn{5}{|c|}{ PenCheck }} \\
\hline & & & & & & & & & & & & & \multicolumn{5}{|c|}{$\beta$-Actin } & & & & & \\
\hline \multicolumn{13}{|c|}{ Pig 1: SMLN-Right } & \multirow{2}{*}{\multicolumn{2}{|c|}{$\begin{array}{l}17.12 \\
19.94\end{array}$}} & & 18.76 & & \multicolumn{5}{|c|}{ Positive } \\
\hline \multirow{2}{*}{\multicolumn{13}{|c|}{ Pig 2: SMLN-Right }} & & & & 18.97 & & & & Positive & & \\
\hline & & & & & & & & & & & & & & & & 18.83 & & & & Positive & & \\
\hline & & & & & 2: SMLN & & & & & & & & & & & 18.81 & & & & Positive & & \\
\hline & & & & & 1: SILN- & & & & & & & & & & & 20.30 & & & & Positive & & \\
\hline & & & & & 1: SILN & & & & & & & & 17 & & & 20.14 & & & & Positive & & \\
\hline & & & & & $2: \mathrm{SILN}$ & & & & & & & & 21 & & & 21.95 & & & & Positive & & \\
\hline & & & & & ig 1: Tor & & & & & & & & & & & 19.23 & & & & Positive & & \\
\hline & & & & & ig 2: Tor & & & & & & & & 22 & & & 20.96 & & & & Positive & & \\
\hline & & & & & ig $1: \mathrm{Spl}$ & & & & & & & & 18 & & & 19.57 & & & & Positive & & \\
\hline & & & & & ig 2: Spl & & & & & & & & & & & 19.00 & & & & Positive & & \\
\hline
\end{tabular}

Whole blood, serum, and swab (nasal, oral, and rectal) samples (A) and different tissue samples (B) collected from two ASFV Nigeria RV502-infected pigs were evaluated for the presence of ASFV genomic material by real-time PCR and to assess the performance of PenCheck ${ }^{\circledR}$ LFA. SMLN: sub-mandibular lymph node; SILN: superficial inguinal lymph node. For (A), the $\beta$-actin Ct value range for all the samples (positive or negative) tested by real-time PCR was 18.76 to 35.78. Pos, positive (green); Neg, negative (yellow); na, not available (gray). 
In addition to the whole blood samples, serum, nasal, oral, and rectal swabs collected from these two animals were tested on PenCheck ${ }^{\circledR}$ LFA. All nasal, oral, and rectal swabs had real-time PCR Ct values above 23; therefore, they tested negative on PenCheck ${ }^{\circledR}$ LFA. The two serum samples, pig \#1, 4 dpi and Pig \#2, $6 \mathrm{dpi}$, with Ct values of 21.01 and 18.74, respectively, tested positive on the PenCheck ${ }^{\circledR}$ LFA. In addition to ante-mortem samples, a number of post-mortem samples (lymph nodes, tonsils, and spleen) collected from pigs \#1 and 2 were tested on the PenCheck ${ }^{\circledR}$ LFA as well. All samples tested positive on PenCheck ${ }^{\circledR}$ LFA and had a high ASFV genome load (Ct values ranging from 17.12 to 22.05) (Table 3 (B)).

To assess the performance of PenCheck ${ }^{\circledR}$ LFA with field samples, we tested whole blood, plasma, and spleen samples collected from domestic pigs on several farms in different geographical regions in Ghana and Nigeria. The samples were first tested for ASFV genomic material using the NCFAD laboratory-based ASFV real-time PCR assay to obtain their Ct values; out of the 50 field samples tested by real-time PCR, a mixture of strong positive, weak positive, and suspicious samples were identified and subjected to testing using PenCheck ${ }^{\circledR}$ LFA. One sample with Ct value lower than 23 unexpectedly tested negative on PenCheck ${ }^{\circledR}$ LFA (Table 4 (A)). Surprisingly, a few samples with Ct values higher than 23 and those deemed suspicious by real-time PCR assay also tested positive on PenCheck ${ }^{\circledR}$ LFA. While the few samples mentioned above did not strictly adhere to the predetermined LOD for PenCheck ${ }^{\circledR}$ LFA, the observations could be due to the degradation of ASFV nucleic acid caused by poor and/or prolonged storage conditions of some of the field samples. For clinical specificity, we also tested 179 known ASFV-negative whole blood samples from Canada (79) and Ghana (100). All the samples were confirmed to be negative for ASFV genomic material by ASFV real-time PCR assay. All the samples except one sample from Ghana tested negative on PenCheck ${ }^{\circledR}$ LFA, demonstrating $99.4 \%$ specificity (Table 4 (B)).

Table 4. Assessing the performance of PenCheck ${ }^{\circledR}$ LFA using field samples.

\begin{tabular}{|c|c|c|c|c|}
\hline \multicolumn{5}{|c|}{ A } \\
\hline \multirow[b]{2}{*}{ Country } & \multirow[b]{2}{*}{ Sample Type } & \multicolumn{2}{|c|}{ Ct Value } & \multirow[b]{2}{*}{ PenCheck } \\
\hline & & ASFV & B-Actin & \\
\hline \multirow{18}{*}{ Ghana (38) } & \multirow{17}{*}{ Whole blood (17) } & 16.13 & 19.79 & Pos \\
\hline & & 22.46 & 25.69 & Neg \\
\hline & & 29.87 & 23.78 & Pos \\
\hline & & 18.14 & 19.85 & Pos \\
\hline & & 19.85 & 21.49 & Pos \\
\hline & & 39.38 & 21.86 & Neg \\
\hline & & 29.76 & 23.88 & Pos \\
\hline & & 20.14 & 21.74 & Pos \\
\hline & & 20.93 & 22.39 & Pos \\
\hline & & 37.86 & 24.90 & Neg \\
\hline & & 39.00 & 26.03 & Pos \\
\hline & & 36.30 & 25.16 & Neg \\
\hline & & 38.85 & 25.45 & Neg \\
\hline & & 35.38 & 26.39 & Neg \\
\hline & & 20.96 & 22.41 & Pos \\
\hline & & 22.50 & 22.76 & Pos \\
\hline & & 20.67 & 24.80 & Pos \\
\hline & Plasma (1) & 22.66 & 21.30 & Pos \\
\hline
\end{tabular}


Table 4. Cont.

\begin{tabular}{|c|c|c|c|c|}
\hline \multicolumn{5}{|c|}{$\mathbf{A}$} \\
\hline \multirow[b]{2}{*}{ Country } & \multirow[b]{2}{*}{ Sample Type } & \multicolumn{2}{|c|}{ Ct Value } & \multirow{2}{*}{ PenCheck } \\
\hline & & ASFV & B-Actin & \\
\hline & \multirow{20}{*}{ Spleen (20) } & 19.32 & 18.95 & Pos \\
\hline & & 25.93 & 18.82 & Pos \\
\hline & & 33.91 & 30.63 & Pos \\
\hline & & 39.15 & 18.21 & Pos \\
\hline & & 26.00 & 18.38 & Pos \\
\hline & & 20.04 & 20.08 & Pos \\
\hline & & 38.99 & 31.68 & Pos \\
\hline & & 18.62 & 18.36 & Pos \\
\hline & & 35.25 & 31.68 & Pos \\
\hline & & 23.33 & 23.76 & Pos \\
\hline & & 34.55 & 33.24 & Pos \\
\hline & & 26.06 & 19.86 & Pos \\
\hline & & 24.19 & 23.24 & Pos \\
\hline & & 31.11 & 29.04 & Neg \\
\hline & & 38.45 & 31.18 & Pos \\
\hline & & 26.20 & 19.02 & Pos \\
\hline & & 32.39 & 28.85 & Pos \\
\hline & & 20.77 & 19.56 & Pos \\
\hline & & 19.47 & 19.95 & Pos \\
\hline & & 26.13 & 21.05 & Pos \\
\hline \multirow{12}{*}{ Nigeria (12) } & \multirow{12}{*}{ Spleen (12) } & 22.74 & 21.83 & Pos \\
\hline & & 22.21 & 21.70 & Pos \\
\hline & & 19.94 & 21.11 & Pos \\
\hline & & 23.25 & 22.13 & Pos \\
\hline & & 19.25 & 25.44 & Pos \\
\hline & & 19.85 & 20.91 & Pos \\
\hline & & 20.99 & 21.60 & Pos \\
\hline & & 22.51 & 20.26 & Pos \\
\hline & & 18.74 & 20.56 & Pos \\
\hline & & 23.81 & 26.57 & Pos \\
\hline & & 23.04 & 26.09 & Pos \\
\hline & & 26.72 & 21.40 & Pos \\
\hline \multicolumn{5}{|c|}{ B } \\
\hline \multirow{2}{*}{\multicolumn{3}{|c|}{ ASFV Real-Time PCR-Negative Samples (No Ct Value) }} & \multicolumn{2}{|c|}{ PenCheck } \\
\hline & & & Negative & Positive \\
\hline & 179 & & $178(99.4 \%)$ & $1(0.6 \%)$ \\
\hline
\end{tabular}

Fifty ASFV real-time PCR-positive field samples (whole blood, plasma, and spleen) collected from Ghana and Nigeria were tested on PenCheck ${ }^{\circledR}$ LFA (A). In addition, a total of 179 ASFV real-time PCR-negative field whole blood samples (with no Ct values) from Ghana (100) and Canada (79) were tested on PenCheck ${ }^{\circledR}$ LFA (B) to assess assay specificity. The $\beta$-actin Ct value range for the 179 ASFV real-time PCR-negative field whole blood samples was 20.25 to 30.17 .

The two discordant field samples (Table $4(\mathrm{~A}, \mathrm{~B})$ ) mentioned above were further tested using a different LFA, INgezim ${ }^{\circledR}$ ASF CROM Ag (Eurofins Technologies Ingenasa, Madrid, Spain), and the results remained unchanged (data not shown). While the real-time PCRpositive, but INgezim ${ }^{\circledR}$ ASF CROM Ag and PenCheck ${ }^{\circledR}$ LFA-negative sample could be explained by poor sample quality, the PenCheck ${ }^{\circledR}$ LFA-positive, but real-time PCR and INgezim ${ }^{\circledR}$ ASF CROM Ag LFA-negative sample could be a true false-positive detection (although with a faint positive test band) by PenCheck ${ }^{\circledR}$ LFA.

To the best of our knowledge, INgezim ${ }^{\circledR}$ ASF CROM Ag is the only other commercially available antibody-based LFA for ASF detection [42]; it targets VP72, a highly immunogenic capsid protein of ASFV, and has shown $100 \%$ specificity. However, it only detected pigs with high viral loads corresponding to $4-7 \mathrm{dpi}$. The assay was recently evaluated for its ability to detect ASFV in whole blood and serum samples collected from experimentally 
infected domestic and wild boars, and wild boars that were naturally infected with highly virulent ASFV strains [43]. The INgezim ${ }^{\circledR}$ ASF CROM Ag detected ASFV in the majority of the whole blood samples collected from experimentally infected domestic pigs and wild boars with $\mathrm{Ct}$ values up to 29 . The assay, however, failed to detect a few blood samples from the experimentally infected animals with $\mathrm{Ct}$ values as low as 20 . When the assay was tested using field-collected wild boar samples, its sensitivity was drastically reduced, likely due to the poor quality of the field samples. The sensitivity of the INgezim ${ }^{\circledR}$ ASF CROM Ag LFA improved when the samples were subjected to a freeze-thaw cycle, possibly owing to the release of ASFV antigens bound to the red blood cells in the sample. However, we observed no difference in our results (sensitivity or specificity) after the freeze-thaw cycle before the samples were tested on the PenCheck ${ }^{\circledR}$ LFA (data not shown). The PenCheck ${ }^{\circledR}$ LFA protocol includes a $5 \mathrm{~min}$ lysis step at room temperature before the sample comes into contact with the lateral flow strip. This may explain the above observation.

In summary, we showed that the commercially available PenCheck ${ }^{\circledR}$ LFA could identify ASFV-infected animals showing a high fever, which coincides with high viral titers that are commonly observed between 3 and 5 dpi with highly virulent ASFV strains. We also showed that PenCheck ${ }^{\circledR}$ LFA could correctly identify most field samples collected from ASFV-infected domestic pigs. In addition to whole blood, the assay can also detect ASFV antigens in other clinical samples such as serum and tissues collected from pigs that succumbed to ASFV infection. Pigs that die of highly virulent ASF infections have high viral loads in their internal organs, such as the spleen, hemorrhagic lymph nodes, and tonsils. Therefore, it is conceivable that PenCheck ${ }^{\circledR}$ LFA would be suitable for detecting ASFV antigens in most of those samples. Owing to low viral loads, sample types such as nasal, oral, and rectal swabs are not suitable for testing using the PenCheck ${ }^{\circledR}$ LFA.

In this study, we only used clinical samples collected from pigs infected with highly virulent ASFV strains. However, using cell culture-amplified ASFV Malta'78 and OURT/88/3 strains, we showed that the PenCheck ${ }^{\circledR}$ LFA is able to detect moderately and low virulent ASFV strains, respectively. Similar to highly virulent strains, moderately virulent ASFV strains also induce high levels of viremia in infected animals, with a slower disease progression. Therefore, it is conceivable that PenCheck ${ }^{\circledR}$ LFA would detect pigs presenting fever and high viremia due to infection with moderately virulent ASFV strains. However, the same cannot be said regarding low virulent ASFV strains as the infected animals seldomly present fever or high viremia; therefore PenCheck ${ }^{\circledR}$ LFA is highly unlikely to detect ASFV in pigs infected with low virulent ASFV strains.

In conclusion, our data showed that, although not as sensitive as molecular assays, the PenCheck ${ }^{\circledR}$ LFA would be a valuable tool for herd-level pen-side detection of ASFV in pigs displaying moderate to severe clinical signs, where access to molecular assays is not feasible. Further improvement of sensitivity of the assay would broaden its use in future ASF control efforts.

\section{Materials and Methods}

\subsection{Lateral Flow Assay}

The PenCheck ${ }^{\circledR}$ LFA kit consists of test strips and test vials containing lyophilized reagents. Whole blood, serum, swabs, and tissues were tested according to the manufacturer's recommendations. Briefly, $200 \mu \mathrm{L}$ of double-distilled water was added to the test vial containing lyophilized reagents, followed by $10 \mu \mathrm{L}$ of the sample. The tube was gently shaken by hand to mix the contents. After $5 \mathrm{~min}$ of incubation at room temperature, the test vial was gently swirled, and the test strip was inserted into the vial. Incubation continued for an additional $20 \mathrm{~min}$, after which it was removed and examined by the naked eye for the appearance of lines. One line (control) indicates a negative sample, and two lines (control and test) indicate a positive sample (Figure S1). 


\subsection{Clinical Samples from Experimentally Infected Animals}

Clinical samples used in this study were obtained from pigs experimentally infected with different ASFV strains at the National Centre for Foreign Animal Disease (NCFAD) or from pigs naturally infected with ASFV field strains in Ghana and Nigeria.

ASFV Georgia 2007/1 (p72 genotype II)-positive whole blood samples were obtained from a recent animal experiment conducted at the NCFAD to evaluate the potential use of oral fluid samples for early detection of ASFV in commercial herds [44]. In this experiment, 1 (pig \#138) out of 24 pigs in the pen was infected with $1 \times 10^{5}$ TCID $_{50}$ of ASFV Georgia $2007 / 1$ (in $1 \mathrm{~mL}$ ) intramuscularly and returned to the pen afterwards. The animals were monitored twice a day for clinical signs, and their rectal temperatures were recorded daily. In addition to pen-based daily oral fluid samples, whole blood, nasal, or oropharyngeal swabs from each animal were collected every other day. To evaluate the PenCheck ${ }^{\circledR}$ LFA, whole blood samples collected from the seeder pig (pig \#138) and 12 other randomly selected contact pigs that developed clinical signs during the early phase of the infection were used.

To obtain additional whole blood and tissue samples, two pigs (pigs 1 and 2) were infected with ASFV RV502, an ASFV p72 genotype II isolate from the 2020 ASF outbreak in Nigeria [45], through the oronasal route $\left(1 \mathrm{X}^{5} 0^{5} \mathrm{TCID}_{50}\right.$ in $3 \mathrm{~mL}, 2 \mathrm{~mL}$ orally, and $0.5 \mathrm{~mL}$ per nostril). After infection, both animals were monitored twice daily for clinical signs and rectal temperatures were recorded daily. In addition to the whole blood samples, different tissue samples (hemorrhagic lymph nodes, tonsils, spleen, and diaphragm) were collected from the two pigs' post-mortem after they succumbed to the infection ( $5 \mathrm{dpi}$ for pig \#1 and $6 \mathrm{dpi}$ for pig \#2). In both of these experiments, four- to five-week-old Landrace-Large White cross-bred grower pigs purchased from a local farm in Winnipeg, Manitoba, Canada, were used.

For specificity testing, three additional strains of cell culture amplified ASFV strains, Malta'78 and OURT /88/3 (p72 genotype I) and Malawi LIL18 / 2 (genotype II), and archived whole blood and spleen samples collected from pigs experimentally infected with three classical swine fever virus (CSFV) strains, Kanagawa (2 pigs), Diepholz (4 pigs), and Koslov (3 pigs), were used. In addition, archived tissue culture-propagated Vesicular stomatitis virus-NJ and IN, foot-and-mouth disease virus-OUKG 11/01 and A24 Cruzeiro, Swine vesicular disease virus UK27/72, and Seneca Valley virus (SVV) were tested.

All ASFV strains used in this study were propagated in primary porcine peripheral leukocytes and tittered on porcine alveolar macrophages as described previously [46].

\subsection{Clinical Samples from Field Outbreaks}

The field samples (all from domestic pigs) used in this study were either obtained as part of an ongoing surveillance program in Ghana or collected during the 2020 ASF outbreak in Nigeria. The whole blood and plasma samples were directly tested as received, while the spleen samples were first homogenized to produce $10 \% \mathrm{w} / \mathrm{v}$ suspensions before testing.

\subsection{Nucleic Acid Extraction and Real-Time Reverse Transcription-Polymerase Chain Reaction}

The total nucleic acids extraction was performed from samples using the MagMAX ${ }^{\mathrm{TM}}$ Pathogen RNA/DNA Kit and the MagMAX Express-96 Magnetic Particle Processor (Life Technologies, Burlington, ON, Canada) following the manufacturer's recommended protocol. The tissue samples used were first homogenized to prepare $10 \% \mathrm{w} / \mathrm{v}$ suspensions before nucleic acid extraction, and the whole blood samples were directly used without further processing.

A previously published TaqMan qPCR assay that specifically amplifies a conserved region of the p72 gene of ASFV was used to quantify the ASFV genomic material [47]. The TaqMan ${ }^{\mathrm{TM}}$ Fast Virus 1-Step master mix (Life Technologies, Burlington, ON, Canada) was used to perform the real-time PCR on Bio-Rad CFX96 Touch ${ }^{\mathrm{TM}}$ (Bio-Rad, Hercules, $\mathrm{CA}, \mathrm{USA})$ using the recommended cycling conditions $\left(50^{\circ} \mathrm{C}\right.$ for $10 \mathrm{~min}, 95^{\circ} \mathrm{C}$ for $3 \mathrm{~min}$, 
followed by 40 cycles of $96{ }^{\circ} \mathrm{C}$ for $3 \mathrm{~s}$ and $60{ }^{\circ} \mathrm{C}$ for $30 \mathrm{~s}$ ). The TaqMan qPCR assay for beta-actin [48] was used as the internal control for optimal nucleic acid extraction and the absence of PCR inhibitors in all assessed samples. Cycle threshold (Ct) values of 35.99 and lower were considered positive, and values between 36 and 40 were deemed suspicious and repeated.

Supplementary Materials: The following supporting information can be downloaded at: https: / / www.mdpi.com/article/10.3390/pathogens11020138/s1. Figure S1. The workflow of PenCheck ${ }^{\circledR}$ LFA testing. To conduct the PenCheck ${ }^{\circledR}$ LFA test, the lyophilized reagents were reconstituted with $200 \mu \mathrm{L}$ of water, followed by the addition of $10 \mu \mathrm{L}$ of the sample. The sample mixture was incubated for $5 \mathrm{~min}$ at room temperature following gentle swirling. After the $5 \mathrm{~min}$ incubation period, the tube was gently swirled, and the test strip was inserted into the vial. The strips were examined by the naked eye for results after $20 \mathrm{~min}$ of incubation at room temperature. One line indicates a negative result, while two lines indicate a positive result. Figure S2. Timeline of the experiment described in Table 2. Pig \#138 (seeder pig) was infected with $1 \times 10^{5}$ TCID $_{50}$ of ASFV Georgia 2007/1 (in $1 \mathrm{~mL}$ ) intramuscularly and immediately returned to the pen containing the naïve contact pigs. The ASFV transmission from the seeder pig to the contact pigs was monitored. The animals were monitored for clinical signs, and their rectal temperatures were recorded daily. Whole blood samples were collected for real-time PCR and PenCheck ${ }^{\circledR}$ LFA analysis on the indicated days.

Author Contributions: Conceptualization, A.A. (Aruna Ambagala); methodology, A.A. (Aruna Ambagala), C.O., and T.O.; software, C.O. and K.N.; validation, C.O. and K.N.; formal analysis, C.O. and A.A. (Aruna Ambagala); investigation, A.A. (Aruna Ambagala) and C.O.; resources, A.A. (Aruna Ambagala), P.D.L., U.H., A.A. (Adeyinka Adedeji), and T.O.; data curation, C.O.; writing-original draft preparation, C.O.; writing-review and editing, C.O. and A.A. (Aruna Ambagala); visualization, C.O. and A.A. (Aruna Ambagala); supervision, A.A. (Aruna Ambagala); project administration, A.A. (Aruna Ambagala); funding acquisition, A.A. (Aruna Ambagala). All authors have read and agreed to the published version of the manuscript.

Funding: Funding for this project was made available through the CFIA A base and the Global Affairs of Canada Project (BIO-2018-0029) on building sustainable diagnostic capability for eradication of ASF in Ghana.

Institutional Review Board Statement: The experiments described here were conducted under the Canadian Council on Animal Care regulations with approval from the Animal Care Committee at the Canadian Science Centre for Human and Animal Health (Animal use document numbers C-19-008 and C-19-012).

Data Availability Statement: The authors will provide the data related to this manuscript upon request.

Acknowledgments: We thank the Animal Care staff at the NCFAD for their assistance during the animal experiments and the technical staff at the mammalian diseases unit for their help in processing the clinical samples. We thank the Vesicular Diseases Unit at the NCFAD for providing us with cell culture-amplified FMDV, SVD, and SVV for specificity testing. Special thanks to Silver Lake Research for providing PenCheck ${ }^{\circledR}$ LFA free of charge for evaluation purposes. We also thank Amy Snow, John Pasick, and Kathleen Hooper-McGrevy at the CFIA for reviewing the manuscript before submission.

Conflicts of Interest: The authors declare no conflict of interest.

\section{References}

1. Sánchez-Vizcaíno, J.; Mur, L.; Gomez-Villamandos, J.C.; Carrasco, L. An update on the epidemiology and pathology of African swine fever. J. Comp. Pathol. 2015, 152, 9-21. [CrossRef]

2. Wang, T.; Sun, Y.; Qiu, H.-J. African swine fever: An unprecedented disaster and challenge to china. Infect. Dis. Poverty 2018, 7, 111. [CrossRef]

3. OIE-World Organisation for Animal Health. African Swine Fever Report. May 10-23, 2019. Available online: /www.oie.int/en/ disease/african-swine-fever/ (accessed on 17 November 2021).

4. Roger, F.; Ratovonjato, J.; Vola, P.; Uilenberg, G. Ornithodoros porcinus ticks, bushpigs, and African swine fever in madagascar. Exp. Appl. Acarol. 2001, 25, 263-269. [PubMed] 
5. WAHID. Office International des Epizooties-World Animal Health Information Database (Wahid) Interface. Available online: https://www.oie.int/en/document/asf_situation_report_3/ (accessed on 19 January 2022).

6. Sánchez Botija, C. Reservorios del virus de la paste porcina Africana. Investigation del virus de la ppa en las arthropodos mediante la prueba de la hemadsocion. Bull. Off. Int. Epizootiol. 1963, 60, 895-899.

7. Plowright, W.; Thomson, G.; Neser, J.; Coetzer, J.; Thomson, G.; Tustin, R. African Swine Fever. Infectious Diseases of Livestock, with Special Reference to Southern Africa; Coetzer, J.A.W., Thomson, G.R., Tutsin, R.C., Eds.; Oxford University Press: Cape Town, South Africa, 1994.

8. Seifert, H.S. Tropical Animal Health; Springer Science \& Business Media: New York, NY, USA, 1996.

9. OIE-WAHIS. 2021. Available online: https:// wahis.Oie.Int/\#/ report-info?Reportid=36844 (accessed on 18 November 2021).

10. Dixon, L.; Escribano, J.; Martins, C.; Rock, D.; Salas, M.; Wilkinson, P. Virus taxonomy. Viiith report of the ictv; Fauquet, C., Mayo, M., Maniloff, J., Desselberger, U., Ball, L., Eds.; Elsevier: London, UK, 2005; pp. 135-143.

11. EFSA Panel on Animal Health and Welfare. Scientific opinion on African swine fever. EFSA J. 2014, 12, 3628. [CrossRef]

12. Montgomery, R.E. On a form of swine fever occurring in british east Africa (kenya colony). J. Comp. Pathol. Ther. 1921, 34, 159-191. [CrossRef]

13. Penrith, M.-L.; Vosloo, W. Review of African swine fever: Transmission, spread and control. J. S. Afr. Vet. Assoc. 2009, 80, 58-62. [CrossRef] [PubMed]

14. Jurado, C.; Fernandez-Carrion, E.; Mur, L.; Rolesu, S.; Laddomada, A.; Sánchez-Vizcaíno, J.M. Why is African swine fever still present in sardinia? Transbound. Emerg. Dis. 2018, 65, 557-566.

15. FAO. African Swine Fever Situation in Asia Update. 2019. Available online: http://www.Fao.Org/ag/againfo/programmes/en/ empres/asf/situation_update.Html (accessed on 17 November 2021).

16. Halasa, T.; Botner, A.; Mortensen, S.; Christensen, H.; Toft, N.; Boklund, A. Control of African swine fever epidemics in industrialized swine populations. Vet. Microbiol. 2016, 197, 142-150. [CrossRef]

17. EFSA Panel on Animal Health and Welfare. Scientific opinion on African swine fever. EFSA J. 2010, 8, 1556. [CrossRef]

18. Callaway, E. Pig fever sweeps across russia. Nature 2012, 488, 565-566. [CrossRef]

19. Sánchez-Cordón, P.J.; Vidaña, B.; Neimanis, A.; Núñez, A.; Wikström, E.; Gavier-Widén, D. 4. Pathology of African swine fever. In Understanding and Combatting African Swine Fever; Wageningen Academic Publishers: Wageningen, The Netherlands, 2021; pp. 87-139.

20. Stear, M. Oie manual of diagnostic tests and vaccines for terrestrial animals (mammals, birds and bees) 5 th edn. Volumes $1 \& 2$. World organization for animal health 2004. Isbn 929044622 6.€ 140. Parasitology 2005, 130, 727.

21. Fu, J.; Zhang, Y.; Cai, G.; Meng, G.; Shi, S. Rapid and sensitive rpa-cas12a-fluorescence assay for point-of-care detection of African swine fever virus. PLoS ONE 2021, 16, e0254815. [CrossRef] [PubMed]

22. Zhang, S.; Sun, A.; Wan, B.; Du, Y.; Wu, Y.; Zhang, A.; Jiang, D.; Ji, P.; Wei, Z.; Zhuang, G. Development of a directly visualized recombinase polymerase amplification-sybr green i method for the rapid detection of African swine fever virus. Front. Microbiol. 2020, 11, 602709. [CrossRef]

23. Ren, M.; Mei, H.; Zhou, M.; Fu, Z.F.; Han, H.; Bi, D.; Peng, F.; Zhao, L. Development of a super-sensitive diagnostic method for African swine fever using crispr techniques. Virol. Sin. 2021, 36, 220-230. [CrossRef]

24. Fan, X.; Li, L.; Zhao, Y.; Liu, Y.; Liu, C.; Wang, Q.; Dong, Y.; Wang, S.; Chi, T.; Song, F.; et al. Clinical validation of two recombinase-based isothermal amplification assays (rpa/raa) for the rapid detection of African swine fever virus. Front. Microbiol. 2020, 11, 1696. [CrossRef]

25. Zhai, Y.; Ma, P.; Fu, X.; Zhang, L.; Cui, P.; Li, H.; Yan, W.; Wang, H.; Yang, X. A recombinase polymerase amplification combined with lateral flow dipstick for rapid and specific detection of African swine fever virus. J. Virol. Methods 2020, 285, 113885. [CrossRef] [PubMed]

26. Miao, F.; Zhang, J.; Li, N.; Chen, T.; Wang, L.; Zhang, F.; Mi, L.; Zhang, J.; Wang, S.; Wang, Y.; et al. Rapid and sensitive recombinase polymerase amplification combined with lateral flow strip for detecting African swine fever virus. Front. Microbiol. 2019, 10, 1004. [CrossRef] [PubMed]

27. Yang, B.; Shi, Z.; Ma, Y.; Wang, L.; Cao, L.; Luo, J.; Wan, Y.; Song, R.; Yan, Y.; Yuan, K.; et al. Lamp assay coupled with crispr/cas12a system for portable detection of African swine fever virus. Transbound. Emerg. Dis. 2021. [CrossRef]

28. Chen, L.; Wen, K.; Chen, F.-E.; Trick, A.Y.; Liu, H.; Shao, S.; Yu, W.; Hsieh, K.; Wang, Z.; Shen, J.; et al. Portable magnetofluidic device for point-of-need detection of African swine fever. Anal. Chem. 2021, 93, 10940-10946. [CrossRef] [PubMed]

29. Tran, D.H.; Tran, H.T.; Le, U.P.; Vu, X.D.; Trinh, T.B.N.; Do, H.D.K.; Than, V.T.; Bui, L.M.; Vu, V.V.; Nguyen, T.L.; et al. Direct colorimetric lamp assay for rapid detection of African swine fever virus: A validation study during an outbreak in vietnam. Transbound. Emerg. Dis. 2021, 68, 2595-2602. [CrossRef]

30. Tao, D.; Liu, J.; Nie, X.; Xu, B.; Tran-Thi, T.-N.; Niu, L.; Liu, X.; Ruan, J.; Lan, X.; Peng, G.; et al. Application of crispr-cas12a enhanced fluorescence assay coupled with nucleic acid amplification for the sensitive detection of African swine fever virus. ACS Synth. Biol. 2020, 9, 2339-2350. [CrossRef] [PubMed]

31. Wang, X.; Ji, P.; Fan, H.; Dang, L.; Wan, W.; Liu, S.; Li, Y.; Yu, W.; Li, X.; Ma, X.; et al. Crispr/cas12a technology combined with immunochromatographic strips for portable detection of African swine fever virus. Commun. Biol. 2020, 3, 62. [CrossRef]

32. Carrio, A.; Sampedro, C.; Sanchez-Lopez, J.L.; Pimienta, M.; Campoy, P. Automated low-cost smartphone-based lateral flow saliva test reader for drugs-of-abuse detection. Sensors 2015, 15, 29569-29593. [CrossRef] [PubMed] 
33. Magambo, K.A.; Kalluvya, S.E.; Kapoor, S.W.; Seni, J.; Chofle, A.A.; Fitzgerald, D.W.; Downs, J.A. Utility of urine and serum lateral flow assays to determine the prevalence and predictors of cryptococcal antigenemia in hiv-positive outpatients beginning antiretroviral therapy in mwanza, tanzania. J. Int. AIDS Soc. 2014, 17, 19040. [CrossRef] [PubMed]

34. Schramm, E.C.; Staten, N.R.; Zhang, Z.; Bruce, S.S.; Kellner, C.; Atkinson, J.P.; Kyttaris, V.C.; Tsokos, G.C.; Petri, M.; Connolly, E.S.; et al. A quantitative lateral flow assay to detect complement activation in blood. Anal. Biochem. 2015, 477, 78-85. [CrossRef]

35. Ang, S.H.; Rambeli, M.; Thevarajah, T.M.; Alias, Y.B.; Khor, S.M. Bioelectronics. Quantitative, single-step dual measurement of hemoglobin a1c and total hemoglobin in human whole blood using a gold sandwich immunochromatographic assay for personalized medicine. Biosens. Bioelectron. 2016, 78, 187-193. [CrossRef]

36. Afonso, C.L.; Alcaraz, C.; Brun, A.; Sussman, M.D.; Onisk, D.V.; Escribano, J.M.; Rock, D.L. Characterization of p30, a highly antigenic membrane and secreted protein of African swine fever virus. Virology 1992, 189, 368-373. [CrossRef]

37. Liu, L.; Luo, Y.; Accensi, F.; Ganges, L.; Rodriguez, F.; Shan, H.; Ståhl, K.; Qiu, H.J.; Belák, S. Pre-clinical evaluation of a real-time pcr assay on a portable instrument as a possible field diagnostic tool: Experiences from the testing of clinical samples for African and classical swine fever viruses. Transbound. Emerg. Dis. 2017, 64, e31-e35. [CrossRef]

38. Daigle, J.; Onyilagha, C.; Truong, T.; Le, V.P.; Nga, B.T.T.; Nguyen, T.L.; Clavijo, A.; Ambagala, A. Rapid and highly sensitive portable detection of African swine fever virus. Transbound. Emerg. Dis. 2021, 68, 952-959. [CrossRef] [PubMed]

39. Zhang, Y.; Li, Q.; Guo, J.; Li, D.; Wang, L.; Wang, X.; Xing, G.; Deng, R.; Zhang, G. An isothermal molecular point of care testing for African swine fever virus using recombinase-aided amplification and lateral flow assay without the need to extract nucleic acids in blood. Front. Cell. Infect. Microbiol. 2021, 11, 131. [CrossRef] [PubMed]

40. Zhao, D.; Liu, R.; Zhang, X.; Li, F.; Wang, J.; Zhang, J.; Liu, X.; Wang, L.; Zhang, J.; Wu, X.; et al. Replication and virulence in pigs of the first African swine fever virus isolated in china. Emerg. Microbes Infect. 2019, 8, 438-447. [CrossRef] [PubMed]

41. Dixon, L.K.; Stahl, K.; Jori, F.; Vial, L.; Pfeiffer, D.U. African swine fever epidemiology and control. Annu. Rev. Anim. Biosci. 2020, 8, 221-246. [CrossRef]

42. Sastre, P.; Gallardo, C.; Monedero, A.; Ruiz, T.; Arias, M.; Sanz, A.; Rueda, P. Development of a novel lateral flow assay for detection of African swine fever in blood. BMC Vet. Res. 2016, 12, 206. [CrossRef]

43. Deutschmann, P.; Pikalo, J.; Beer, M.; Blome, S. Lateral flow assays for the detection of African swine fever virus antigen are not fit for field diagnosis of wild boar carcasses. Transbound. Emerg. Dis. 2021, 1-5. [CrossRef]

44. Goonewardene, K.; Chung, C.J.; Goolia, M.; Blakemore, L.; Fabian, A.; Mohamed, F.; Nfon, C.; Clavijo, A.; Dodd, K.A.; Ambagala, A. Evaluation of oral fluid as an aggregate sample for early detection of African swine fever virus using four independent pen-based experimental studies. Transbound. Emerg. Dis. 2021, 68, 2867-2877. [CrossRef]

45. Adedeji, A.J.; Luka, P.D.; Atai, R.B.; Olubade, T.A.; Hambolu, D.A.; Ogunleye, M.A.; Muwanika, V.B.; Masembe, C. First-time presence of African swine fever virus genotype ii in nigeria. Microbiol. Resour. Announc. 2021, 10, e00350-21. [CrossRef]

46. Onyilagha, C.; Nash, M.; Perez, O.; Goolia, M.; Clavijo, A.; Richt, J.A.; Ambagala, A. Meat exudate for detection of African swine fever virus genomic material and anti-asfv antibodies. Viruses 2021, 13, 1744. [CrossRef] [PubMed]

47. Tignon, M.; Gallardo, C.; Iscaro, C.; Hutet, E.; Van der Stede, Y.; Kolbasov, D.; De Mia, G.M.; Le Potier, M.-F.; Bishop, R.P.; Arias, M.; et al. Development and inter-laboratory validation study of an improved new real-time pcr assay with internal control for detection and laboratory diagnosis of African swine fever virus. J. Virol. Methods 2011, 178, 161-170. [CrossRef] [PubMed]

48. Duvigneau, J.C.; Hartl, R.T.; Groiss, S.; Gemeiner, M. Quantitative simultaneous multiplex real-time pcr for the detection of porcine cytokines. J. Immunol. Methods 2005, 306, 16-27. [CrossRef] 